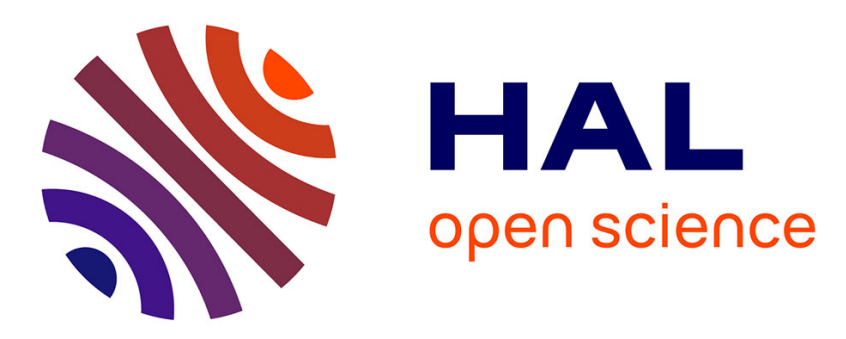

\title{
Pseudo-haptic feedback: can isometric input devices simulate force feedback?
}

Anatole Lécuyer, Sabine Coquillart, Abderrahmane Kheddar, Paul Richard, Philippe Coiffet

\section{- To cite this version:}

Anatole Lécuyer, Sabine Coquillart, Abderrahmane Kheddar, Paul Richard, Philippe Coiffet. Pseudohaptic feedback: can isometric input devices simulate force feedback?. VR: Virtual Reality, Mar 2000, New Brunswick, NJ, United States. pp.83-90, 10.1109/VR.2000.840369 . hal-00844954

\section{HAL Id: hal-00844954 https://hal.science/hal-00844954}

Submitted on 2 Oct 2019

HAL is a multi-disciplinary open access archive for the deposit and dissemination of scientific research documents, whether they are published or not. The documents may come from teaching and research institutions in France or abroad, or from public or private research centers.
L'archive ouverte pluridisciplinaire HAL, est destinée au dépôt et à la diffusion de documents scientifiques de niveau recherche, publiés ou non, émanant des établissements d'enseignement et de recherche français ou étrangers, des laboratoires publics ou privés. 


\section{Pseudo-Haptic Feedback : Can Isometric Input Devices Simulate Force Feedback?}

\author{
Anatole Lécuyer* \\ Aerospatiale Matra CCR
}

\author{
Sabine Coquillart ${ }^{\dagger}$ \\ INRIA Rocquencourt
}

\author{
Abderrahmane Kheddar \\ CEMIF-SC, UEVE
}

\author{
Paul Richard \\ LRP, CRIIF
}

\author{
Philippe Coiffet \\ CNRS, LRP
}

\begin{abstract}
This paper considers whether a passive isometric input device, such as a Spaceball ${ }^{T M}$, used together with visual feedback, could provide the operator with a pseudo-haptic feedback.

For this aim, two psychophysical experiments have been conducted. The first experiment consisted of a compliance discrimination, between two virtual springs hand-operated by means of the Spaceball ${ }^{T M}$. In this experiment, the stiffness (or compliance) JND turned out to be 6\%. The second experiment assessed stiffness discrimination between a virtual spring and the equivalent spring in reality. In this case, the stiffness (or compliance) JND was found to be $13.4 \%$.

These results are consistent with previous outcomes on manual discrimination of compliance. Consequently, this consistency reveals that the passive apparatus that was used can, to some extent, simulate haptic information.

In addition, a final test indicated that the proprioceptive sense of the subjects was blurred by visual feedback. This gave them the illusion of using a non isometric device.
\end{abstract}

\section{Introduction}

Isotonic or isometric $3 \mathrm{D}$ input devices ${ }^{1}$ are clever devices for the purpose of 3D interactions and 3D manipulations of objects. They are compatible with almost all ma-

\footnotetext{
*Corresponding author: Anatole Lécuyer. Aerospatiale Matra, Centre Commun de Recherche Louis Blériot, 12 rue Pasteur, 92152 Suresnes, France. Email: anatole.lecuyer@aeromatra.com or anatole.lecuyer@inria.fr

†Corresponding author: Sabine Coquillart. INRIA, Domaine de Voluceau, Rocquencourt - BP 105, 78153 Le Chesnay Cedex, France. Email: sabine.coquillart@inria.fr

${ }^{1}$ Zhai classified the input devices into two categories : ISOMETRIC devices (they offer resistance and stay put while you exert force on them) and ISOTONIC devices (they offer no significant resistance and are used to track users as they move around the virtual world) [21].
}

jor CAD softwares available on the market. Their userfriendliness has shown their potential usability as a tool for off-line robot programming and teleoperation, or virtual prototyping. For instance, to teleprogram the Mars Pathfinder Sojourner robot, the operator uses a Spaceball ${ }^{\mathrm{TM}}$ as an input tool along with a virtual reality interface [1]. The Spaceball ${ }^{\mathrm{TM}}$ is an isometric input device with six degrees of freedom (dof) which is now commercialized by the Spacetec company [3]. The Magellan SpaceMouse ${ }^{\mathrm{TM}}$ [2] - another 6dof isometric device - was successfully used by the DLR - the German Space Agency - operators and astronauts to teleoperate a space robot within the context of the well-known ROTEX experiment [11].

Since isometric or isotonic input interfaces are completely passive, they have never been regarded as being able to return forces. How the use of the properties of an isometric input device, the Spacetec Spaceball ${ }^{\mathrm{TM}}$ 2003C model, together with visual feedback to provide force information to the operator is the subject of the following paragraph.

To begin with, there is to take advantage of the mechanical characteristics of the isometric device : its internal stiffness and its thrust. Those characteristics are combined with visual feedback to provide a kind of pseudo-force feedback. For example, let us assume that one manipulates a virtual cube in a 3D virtual environment (VE). The cube must be inserted inside a narrow duct. As the cube penetrates the duct, its speed is reduced. In other words, the Spaceball ${ }^{\mathrm{TM}}$ output resolution, which controls the cube motion, is decreased. Consequently, the user will instinctively increase its pressure on the ball which results in the feeding back of an increasing reaction force by the static device. The coupling between the slowing down of the object on the screen and the increasing reaction force coming from the device gives the user the illusion of a force feedback as if a friction force between the cube and the duct was directly applied to him.

This "illusion" of force feedback was first qualitatively estimated with a group of 18 people during an experiment 
called the swamp. The subjects were told to manipulate a virtual cube, displayed on the horizontal plane, and to cross square areas (see Figure 1). When over these areas, the speed of the cube was either accelerated or slowed down. The subjects were told to describe their sensations when the cube was crossing the areas, and to compare the sensations they felt when using either the Spaceball ${ }^{\mathrm{TM}}$ or a classical 2D mouse. While using the Spaceball ${ }^{\mathrm{TM}}$, their accounts re-

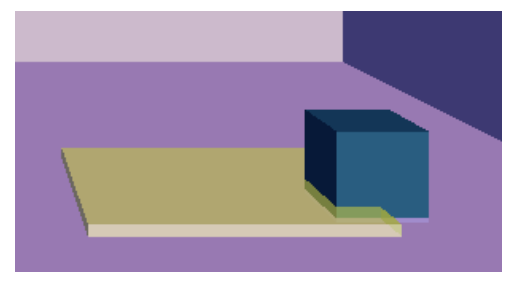

\section{Figure 1. Swamp experiment: cube crossing a slowing down area.}

vealed that the subjects felt "something" as the cube crossed these areas. Most subjects experienced a sense of friction, gravity or viscosity when the cube's movement was slowed down. They found that forces were much more perceptible with the Spaceball ${ }^{\mathrm{TM}}$ than with the $2 \mathrm{D}$ mouse. The perception of forces was a bit less sharp when the cube was accelerated. This is probably due to the fact that the reactive force from the Spaceball ${ }^{\mathrm{TM}}$ is more perceptible during compression phases.

Those qualitative indications revealed the potentialities of this concept, but they did not measure or identify the characteristics of such an illusion. It was necessary to evaluate the feedback more quantitatively. A compliance discrimination task between a real and a virtual spring was chosen as a simple evaluation task. The real spring was tested first in the real environment (RE), then the virtual spring was tested in the VE. The virtual spring was graphically displayed on a computer screen and was dynamically animated when pushing the Spaceball ${ }^{\mathrm{TM}}$ ball. If the Spaceball ${ }^{\mathrm{TM}}$, used together with visual feedback, allows one to discriminate a virtual spring stiffness from a real one, then the whole system may thus be fit for feeding haptic information which was supposedly difficult to provide without a force feedback interface.

First, an overview of previous work in the field of 3D input interfaces evaluation and sensory illusions will be presented. It will be followed by a description of the experimental system which was set up for the evaluation of the stiffness of the real and virtual springs. Previous works concerning compliance or stiffness discrimination and JND (Just Noticeable Difference) will also be mentioned. The two following sections describe two psychophysical exper- iments: the compliance discrimination between two virtual springs, and the compliance discrimination between a virtual spring and a real one. The first experiment is a VE evaluation; while the second experiment is the main subject of the study. The paper ends with a general conclusion and a reference to further work.

\section{Previous work}

Force/tactile interfaces have been developed in recent years [6] in order to provide force/touch feedback to users. They receive motor actions from the user and send haptic images to him. These interfaces are used to simulate a wide range of object dynamics such as hardness and elasticity. Yet, today, they are still expensive and complex.

The perception of real or virtual environments is not restricted to the intra-sensory interpretation cues. Cues sent by different senses are somehow interpreted together. For instance, manipulating objects combines tactile, kinesthetic sensations and often vision [7]. Given these complexities, it would seem more appropriate to investigate the "pluralistic" nature of sensory perception, rather than one isolated sense. Aldridge [4] observed that the visual representation of a virtual object has some effect on the integration of the touch feedback. He stated that further experiments needed to be carried out in order to explore the extent of such "visual dominance".

Previous work on visual dominance showed that multiple cues offer a high level of redundancy and can improve signal-to-noise ratios. For instance, it has been shown that lip-reading modifies the auditory cortex, and enhances auditory perception [14]. One interesting issue is how these different sources of information are all combined to form what might be called holistic perceptions. A famous example of visual dominance is the Ventriloquist's effect [19]. Diderot (1749), offered early support for the existence of sensory dominance [13]. Several researchers have demonstrated a dominance of vision over taction [7]. Lee and Lishman provided evidence that vision plays an integral role in human stance control (balance). This "visual proprioceptive control" is shown to dominate over non-visual information. Lee and Lishman described also the tuning role that visual proprioception plays in learning a new stance (i.e. ankle-foot proprioception). This suggests that vision plays a major role in making things feel the way they do.

But vision may sometimes make things feel different than they are. Katz [7] observed that different materials (paper, rubber, leather, etc..) can easily be interpreted differently by blindfolded subjects. Srinivasan [16] found that vision could also mislead someone during a compliance discrimination task between two springs. The displacement of the springs was visually observed on a computer screen, while springs were pressed manually by means of a me- 
chanical apparatus. Srinivasan observed that an inapropriate vision feedback can totally invert the stiffness perception and the result of the discrimination, which ushers in the illusion concept.

Illusion plays a central role in a VE perception. Illusion is a non veridical perception, a mistake made by the brain and not by our senses. Well-known optical illusions such as the Müller-Lyer illusion are extensively described in scientific works [10]. Some haptic illusions may also be revealed by simple experiments. For example, Weber first observed that the temperature of an object influences the haptic perception of its weight: a cold coin seems heavier than the same coin when warmer [15]. Another haptic illusion is the size-weight illusion: a large radius ball seems heavier than a ball of the same weight, but with a smaller radius. Recently, Ellis and Lederman [8] established the size-weight illusion as a primarily haptic phenomenon, despite its having been more traditionally considered an example of vision influencing haptic processing. The resort to intra and intersensory illusions and dominance can be relevant when used in VR applications. The following paper concentrates on the use of these potentialities.

\section{Experimental set-up}

As already stated, the aim of the experimental system described is to measure the capacity to feed back haptic information by means of a passive isometric input device and vision feedback, which is the key issue of the scheme. It is coupled with the force applied on the Spaceball ${ }^{\mathrm{TM}}$, and any change in the visual feedback generates a difference in force perception. The perception of the stiffness of the spring involves a multi-modal combination of force and displacement. Thus, it seemed to be an appropriate model to demonstrate the general concept.

An experiment of compliance discrimination of springs was then chosen because of the simplicity of the model of stiffness, relevant previous works on manual discrimination of compliance, and the fact that springs are a classical and fundamental element in computer graphics and computer haptics modeling. The stiffness discrimination lies between a virtual spring and a real one. In the VE case, the spring displacement is visually displayed on a computer screen. The force information of the spring stiffness is inherent to the reactive force from one's interaction with the Spaceball ${ }^{\mathrm{TM}}$. Those two independent sensory cues (virtual displacement motion and reactive force of the Spaceball ${ }^{\mathrm{TM}}$ ) should allow the user to discriminate the stiffness of a virtual spring from that of a real one. If this works out, the concept is apt to provide haptic information which was a priori not to be simulated without an actual force feedback device. Real springs are tested in RE conditions. Each spring looks like a trumpet piston (see Figure 2). Three real springs were

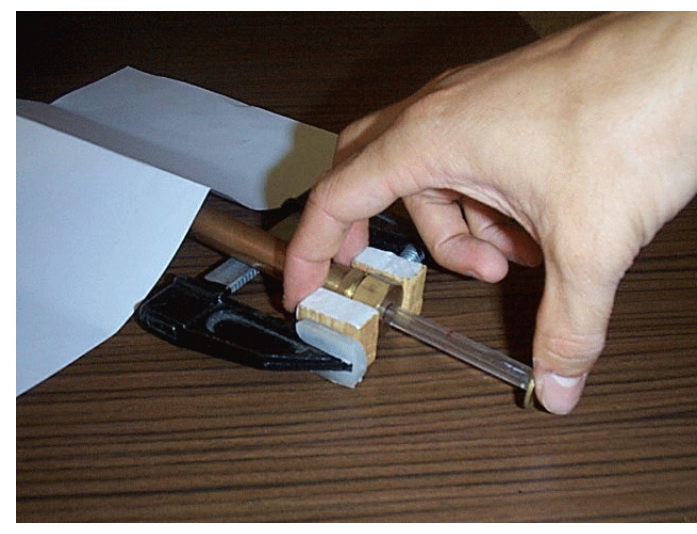

Figure 2. Real spring embedded in a piston

used with different degrees of stiffness: 249,363 and 544 $\mathrm{N} / \mathrm{m}$. Their stiffness was empirically derived by measuring spring displacement when fixed weights were applied on each of them. Friction effects inside the real piston were nearly canceled by directly applying lubricant on the spring and inside the static part of the piston.

The visual display of the virtual spring is fundamental: spatial reference must be the same one when comparing the virtual spring motions and the real ones. The virtual spring is thus visually displayed on a monoscopic workstation screen, as similar as possible to the real one (see Figure 3 ), and of the same size as the real spring. Special attention was given to many graphical features (color, texturing, etc.) in order to recreate the virtual piston with the highest possible realism. A Spaceball ${ }^{\mathrm{TM}}$ ball was also rendered on the left side of the screen to facilitate the comprehension of the scale factor between VE and RE.

The displacement of the virtual spring $D_{\text {virtual }}$ is deduced from the force applied by the user $F_{\text {user }}$ using the wellknown equation 1 , in which $K_{\text {virtual }}$ is the virtual spring stiffness. The force applied by the user on the ball is monitored by internal Spaceball ${ }^{\mathrm{TM}}$ sensors. The Spaceball ${ }^{\mathrm{TM}}$ (force applied by user )/(sensors output) profile was manually identified with a dynamometer. A maximum $10 \%$ uncertainty in the output data was observed. A maximum pushing limit is indicated on the virtual display by a red mark on the moving part of the piston. It corresponds to the sensing limit of the Spaceball ${ }^{\mathrm{TM}}$ 's force sensors in the case of the stiffest virtual spring. The red mark is also printed on the real spring to keep the same visual aspect.

$$
D_{\text {virtual }}=F_{\text {user }} / K_{\text {virtual }}
$$

In order to obtain similar tactile and grasping sensations in the real and virtual cases, the same moving part of the piston was fixed on the Spaceball ${ }^{\mathrm{TM}}$, by means of two plastic links (see Figure 4). The grasping of the virtual spring is thus 
similar to the real one, thanks to the plastic upper link on the Spaceball ${ }^{\mathrm{TM}}$ on which the subject can put his forefinger and his middle finger (see Figure 4).

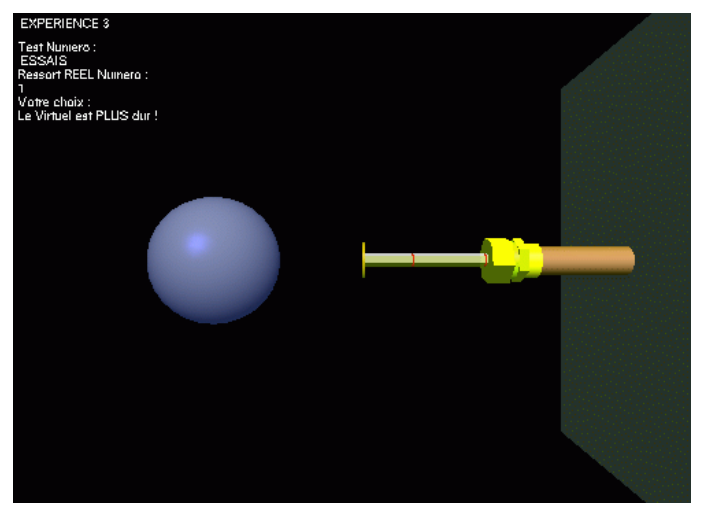

Figure 3. Visual display of a virtual spring

Finally, for the testing of the real spring, subjects grasp the real piston as shown in Figure 2, and push the moving part of the piston with an active motion of the thumb. For the testing of the virtual spring, the subject applies a force on the Spaceball ${ }^{\mathrm{TM}}$ by pushing the moving part of the piston fixed on the Spaceball ${ }^{\mathrm{TM}}$ 's base with the thumb (see Figure 4). He/she looks at the screen so as to see the displacement of the virtual spring resulting from his/her actions.

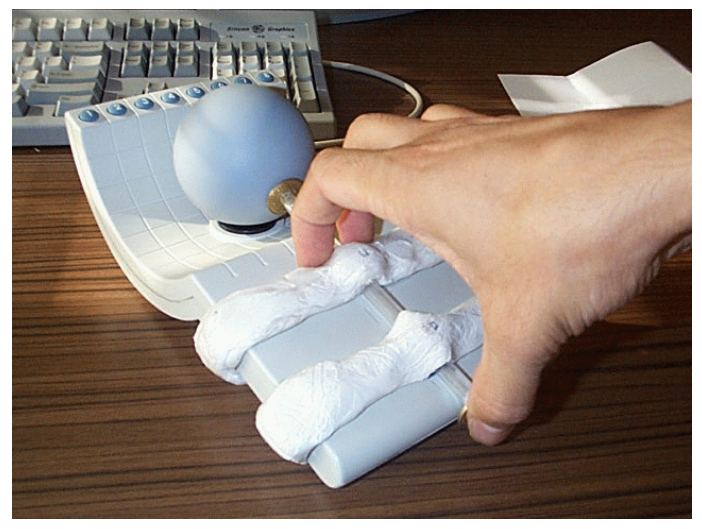

Figure 4. "Modified" isometric device

\section{Previous work on compliance discrimination}

In the RE case, the compliance discrimination has been widely studied. The Just Noticeable Difference (JND) is the just detectable increment (or decrement) of intensity for a specific stimulus. Jones and Hunter [12] found that the compliance discrimination JND in forearms was $23 \%$ of the intensity of the stimulus. Tan studied the manual discrimination of the compliance with active motion of the finger by using an electromechanical apparatus called the 'linear grasper'. She first found a JND of $8 \%$ in the case of a fixed squeezing distance [18]. But after reducing terminal force cues by using a roving squeezing distance, the JND reached up to $22 \%$ [18]. When the mechanical work cues were also eliminated through an equal-work-force-displacement profile, the JND was found to vary between $15 \%$ and $99 \%$ [17].

In the VE case, Tzafestas found a JND of $44 \%$ with the help of the LRP dextrous hand master which is an exoskeleton glove with 14 active dof [20]. The virtual discrimination was made between two virtual balls displayed on a computer screen. The balls were pressed alternatively with the thumb and the forefinger of the master glove.

As far as the authors of this paper know, no studies on compliance discrimination have ever been carried out using simultaneously a real spring and a virtual one.

\section{Compliance discrimination between two vir- tual springs}

It seems at first that the experimental set-up presents many uncertainties linked to the identification of the Spaceball ${ }^{\mathrm{TM}}$ 's force/output profile, graphical approximations, manual evaluation in $\mathrm{RE}$ of the stiffness of the springs, small differences in graspings and frictions between RE and VE. Therefore the reliability of the VE has to be taken into account first. In order to evaluate the virtual model of the spring, a compliance discrimination experiment between two virtual springs is first carried out.

\subsection{Experimental procedure}

4 people, from the age of 21 to 38 , took part in this experiment. There were 3 men and 1 woman with no known perception disorders. All the subjects were right-handed and used their dominant hand to perform the grasping task.

The psychophysic method used was a constant stimuli method with a forced choice and (+,-) paradigm (see [9] for a description of the method). During each trial the subject had to choose between two virtual springs displayed on the same computer screen and to say which one of the two was the stiffer.

Three values of virtual reference stiffness were used: 249,363 and $544 \mathrm{~N} / \mathrm{m}$. Each spring was compared with five possible stiffer springs whose stiffness varied from the reference stiffness by $+0,+5,+10,+15$ and +20 percent. Each subject tested all the possible pairs. For each subject each pair appeared 25 times in random order. The total amount of trials was then 100 a pair and the total amount of trials was 1500. For each trial the reference stiffness was randomly 
associated either with the top spring or with the bottom one on the screen. During each trial the subject had the possibility to test each spring as many times as he wanted to, but he was asked to answer as fast as possible. No response feedback was given after each trial. When testing a virtual spring subjects were told not to go beyond the red mark printed on the moving part of the piston.

\subsection{Results and discussion}

The analysis of the results used to determine the differential threshold can be found in [9] and [5]. The Weber fraction is a common parameter used to evaluate the performance of the discrimination. The Weber fraction is the JND divided by stimulus intensity. It is sometimes assimilated to JND in literature ([17], [12]). The average Weber fraction found for compliance discrimination between two virtual springs with our system is of $6 \%$ (see Figure 5). The

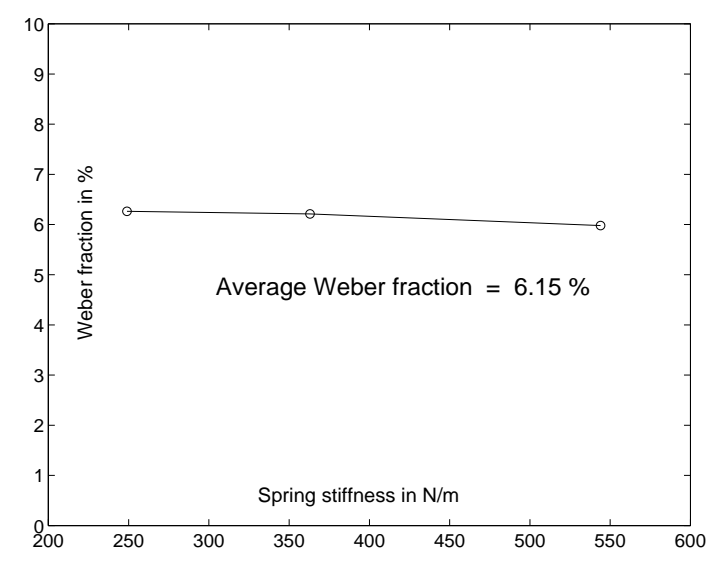

Figure 5. Weber fraction for compliance discrimination of virtual springs

red mark drawn on the piston to represent the maximum squeezing limit is considered a terminal force cue for subjects. The conditions of the experiment are similar to Tan's first experiment -i.e. with a constant squeezing distancewhen she found a JND of $8 \%$ [18]. The JND discrimination between virtual springs seems then consistent with previous works addressing manual compliance discrimination, although the discrimination has been made within a VE with uncoupled sensory information. Furthermore all subjects found that the model of the spring was visually and haptically realistic.

The result of this experiment indicates that the configuration of this system can simulate the model of a virtual spring realistically. However, a compliance discrimination in a VE can be performed with most of other input devices, without force feedback, but by using sound or visual substi- tutions as force cues. It is therefore necessary to study the concept with a task which could not be performed, or so it seems, without using a force feedback device.

\section{Compliance discrimination between a real spring and a virtual one}

The test that was chosen is still a compliance discrimination task, but between a real spring and a virtual one in this case. Since isotonic input devices do not return real force information, this task should not be achievable with isotonic 2D mice, or joysticks without force feedback. With the adopted apparatus, the discrimination task is still not achievable if one simply presses the Spaceball ${ }^{\mathrm{TM}}$ with closed eyes. Obviously this is due to the fact that there will be no feedback of displacement information. Neither is it possible when one stops touching the Spaceball ${ }^{\mathrm{TM}}$, since he/she no longer receives force information.

\subsection{Experimental procedure}

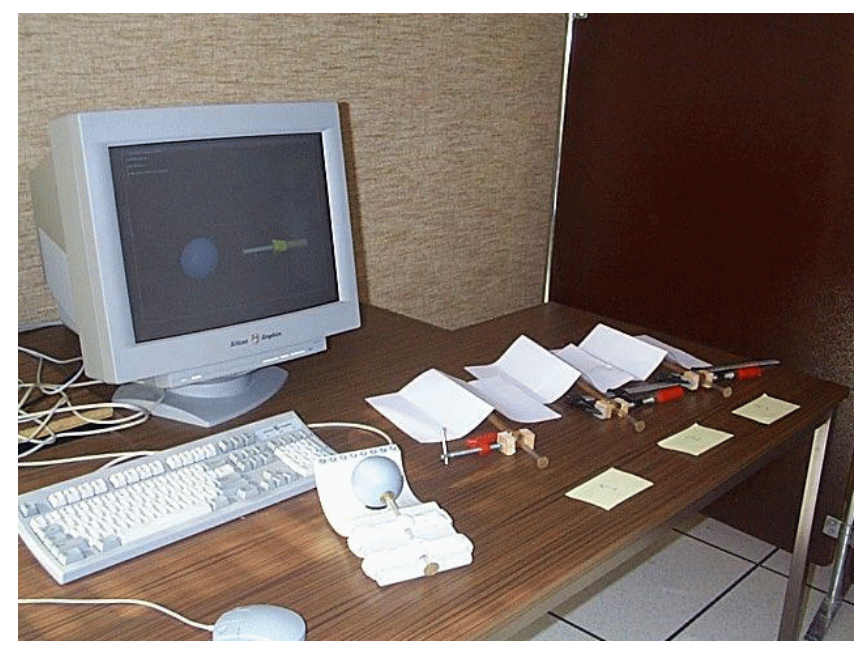

Figure 6. Overview of the experimental set up

27 people, from the age of 21 to 63 , took part in this experiment. There were 20 men and 7 women with no known perception disorders. Among them, 3 men were lefthanded. All the subjects used their dominant hand to perform the grasping task. The psychophysic method used was also a method of constant stimuli with a forced choice and a $(+,-)$ paradigm. During each trial the subject first had to test the stiffness of a real spring (the reference stimulus), and then, to test the stiffness of a virtual spring (the comparison stimulus). The testing and grasping of real and virtual 
springs were performed as described in Section 3. After investigating the stiffness stimuli of both the virtual and real springs, the subject had to tell whether the virtual spring was more or less stiff than the real spring of reference (see Figure 6).

Three real springs were used with stiffness of 249,363 and $544 \mathrm{~N} / \mathrm{m}$. Each real spring was compared with twelve possible virtual springs whose stiffness varied from the reference RE stiffness by: $-40,-30,-20,-10,0,+10,+20,+30$, $+40,+50,+60$ or +70 percent. Each subject tested all the possible pairs. For each subject each pair appeared only once in random order. The total amount of trials was then 27 a pair and the total amount of trials was 972 . During each trial, the subject had the possibility to go from one spring to the other without time limit. No response feedback was given after each trial When testing the virtual spring subjects were asked not to go beyond the red mark printed on the moving part of the piston. But in the RE case subjects had no restrictions concerning the pushing of the piston and their going beyond the red mark.

\subsection{Results}

The analysis of the results follows the classical method described in [9] and [5]. The proportion (or probability) of "the virtual spring is stiffer" answers when the virtual spring is compared to the real one is used to trace the psychometric function concerning the three values of stiffness (see Figure 7). Assuming that these functions correspond to normal distributions, the z-score transformation of the probability of "stiffer" answers will become a linear function (see Figure 8). On Figure 8, the best fitting straight lines are found through the least square method. A z-score equal to 0.67

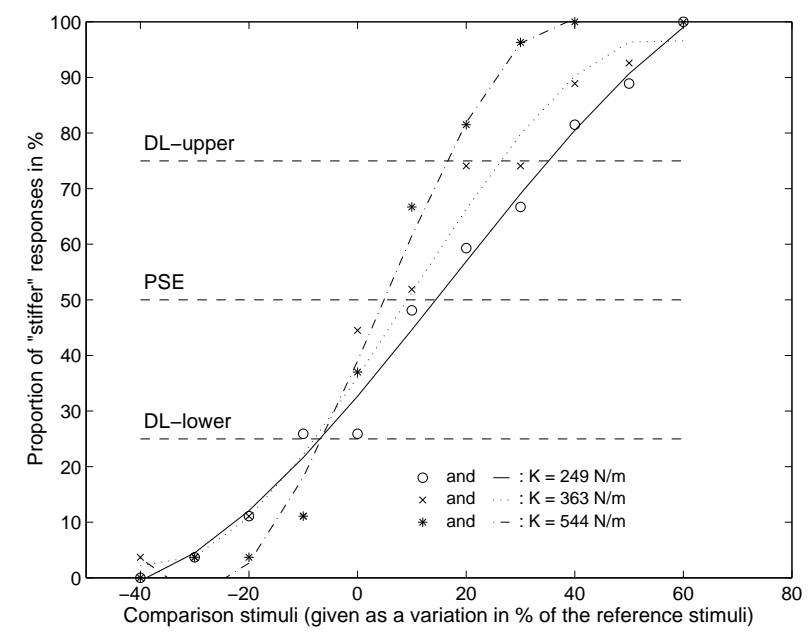

Figure 7. Psychometric functions

(probability of 75\%) is generally associated with the upper Differential Limen (DLu) and a z-score equal to -0.67

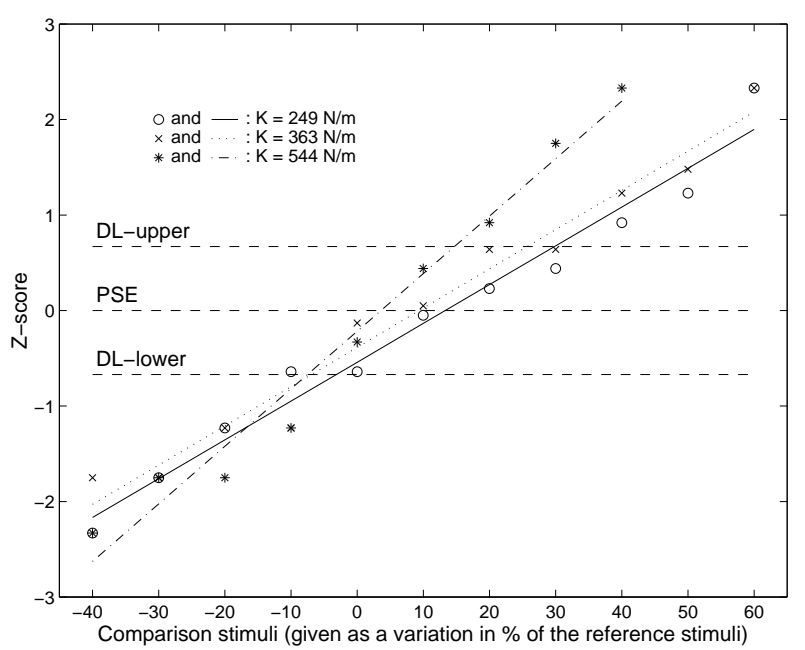

Figure 8. Z-score transformations

(probability of 25\%) is generally associated with the lower Differential Limen (DLl). The Point of Subjective Equality (PSE) represents the value of the compared stimulus subjectively perceived as being equal to the stimulus of reference. In most cases, the PSE does not correspond exactly to the physical value of the standard reference stimulus. PSEs are given by a z-score equal to 0.0 (probability of 50\%) and are displayed on Figure 9. The Weber fraction is given by equation 2 . The Weber fraction for the three values of stiffness are shown on Figure 10. The resulting average Weber fraction $\mathcal{W}$ is equal to $13.4 \%$.

$$
\mathcal{W}=(D L u-P S E) / P S E
$$

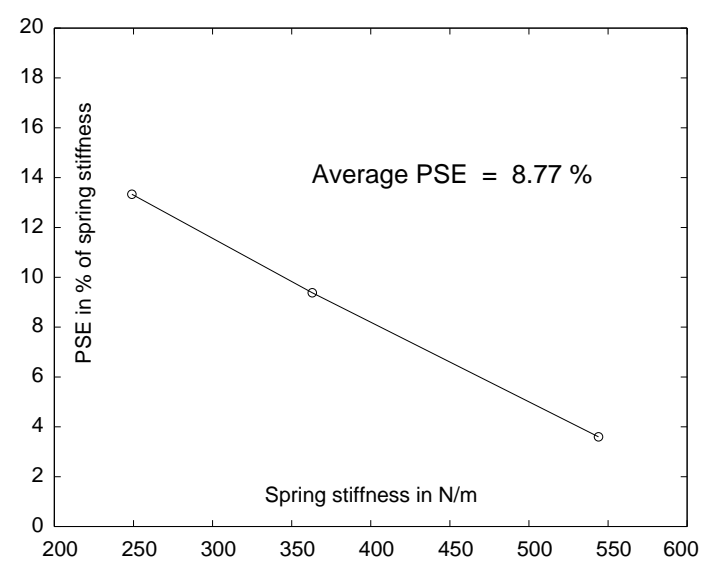

Figure 9. PSE variation 


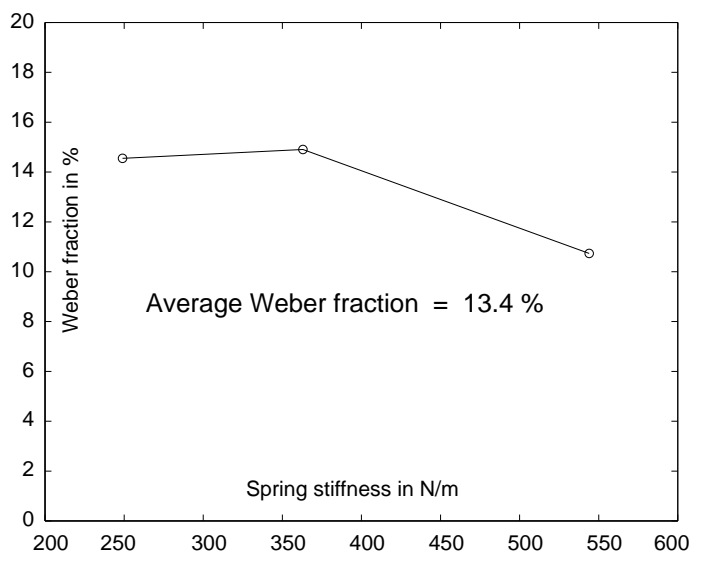

\section{Figure 10. Weber fraction for compliance dis- crimination between a virtual spring and a real one}

\subsection{Discussion}

At the end of the experiment, the subjects were asked to comment upon their cognitive strategies when evaluating the virtual and the real springs, and to tell whether they used the red marks or not, as they sometimes used them and sometimes did not pay any attention to them. The answers make it difficult to know whether the red mark was always used as a terminal force cue for the virtual/real spring comparison or not. The $13.4 \%$ JND should thus be compared with $8 \%$ and $22 \%$ JNDs previously found by Tan in fixed and roving squeezing distance experiments [18]. The herein result seems consistent with previous studies concerning manual discrimination of compliance. Nevertheless all previous experiments were carried out within a single environment while in this experiment, the transition between the RE and the VE is also to be taken into account and has probably had a negative influence on the result of the performance.

The PSE represents the subjective displacement of the reference stimulus during the bilateral transitions from the RE to the VE. The PSE difference from the reference stimulus is always positive and it decreases monotonically with stiffness and thus with maximum displacement of the thumb (see Figure 9). Consequently the subjects have a tendency to underestimate the virtual spring. The less stiff the reference spring is, the more underestimated the comparison virtual spring is. Many reasons could account for this phenomenon:

- Some psychological effect stemming from the bilateral transitions between the VE and the RE and a lack of confidence in the virtual spring, or perhaps as well as a problem of sensory memory persistence generating a negative error upon the reference stimuli during the transient phase of adaptation to VE.

- Another explanation could be the contradiction between the proprioceptive feeling of motion of the thumb and the visual feedback of the displacement of the spring. The thumb is nearly static when pressing the Spaceball ${ }^{\mathrm{TM}}$ while the spring moves extensively on the screen, especially in the case of the less stiff spring. This makes for a phenomenon of sensory dominance: the visual feedback replaces the proprioceptive sense to some extent. The visual feedback must be assimilated by the brain to assess the displacement of the spring and the mechanical work, which is another important factor in compliance evaluation [17]. But the studies on the transfer from RE to VE and on sensory substitution [16] [7] fail to conclude whether visual feedback can or cannot compensate for both mechanical work and displacement cues within the context of this study.

\section{Conclusion}

At the beginning of the experiment described above, there were many uncertainties concerning the feasibility of a comparison between a virtual and a real physical model because of the difficulty of the bilateral transition between $\mathrm{RE}$ and VE and problems related to the verisimilitude of the virtual spring model. But with the apparatus that was set up, subjects were able to discriminate successfully between a virtual spring and a real one with a JND of $13.4 \%$. This JND value is consistent with previous works on manual compliance discrimination [18] [17] [12]. It shows the possibility to feed back haptic information to the user without using a force feedback device but simply by combining a passive isometric input device with a visual feedback. This may remain impossible when using an isotonic input interface.

The experiment is based on the coupling of the visual feedback and the internal isometric device resistance which naturally reacts to the user's applied force. The overall system returns a force cue called pseudo-haptic feedback. This pseudo-haptic feedback will probably not replace an actual haptic one but can be useful for some simple simulations, making full use of the 6 possible degrees of freedom of isometric input devices.

The most surprising result is the substitution of the proprioceptive information at the level of the thumb by the visual feedback . All subjects were able to discriminate between a real spring and a virtual one without the proprioceptive information from the thumb since the displacement during the virtual spring evaluation was very small. Furthermore, at the end of the experiment, the last 10 subjects were asked to draw a line segment corresponding to the maximum displacement of their thumb when pressing a virtual spring. The result (see Figure 11) indicates an 
overestimation of the displacement of their thumbs varying from 2 to 8 times the actual displacement, with an average overestimation of 5 times, which means that the illusion of their proprioceptive sense is strong- as if they perceived the Spaceball ${ }^{\mathrm{TM}}$ as a non isometric device.

The experiment relies on the "illusion" concept which could be used in many more VR applications. The visual dominance was used to influence the perception of the displacement of a virtual spring. Indeed, people found that the virtual spring model was realistic. This virtual spring constitutes a multi-modal virtual object whose perceptive cues are uncoupled and provided by different sensory modalities on the haptic and visual modes.

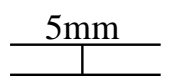

Spaceball maximum displacement

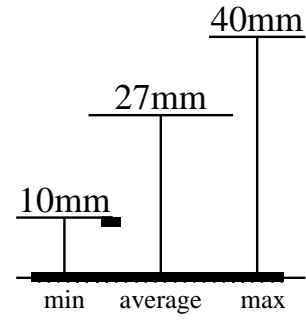

Maximum thumb displacement perceived

\section{Figure 11. Thumb displacement perceived}

Future work deals with the evaluation of pseudo-haptic use of isometric interfaces. First, further evaluation of the pseudo-haptic feedback through different perceptual tasks should be performed such as discrimination of the weight of objects. Secondly, a comparison with force feedback devices is also necessary to position the potential of pseudo-haptic feedback. Finally, applications of pseudohaptic feedback on industrial tasks remain to be defined, particularly when resorting to the 6 degrees of freedom of the Spaceball ${ }^{\mathrm{TM}}$. A performance experiment will be set up to measure if this illusion can be used to improve performance in an industrial task.

\section{Acknowledgements}

The authors would like to thank the Spacetec company for providing freely the Spaceball model of this experiment. They would also like to thank all the subjects who took part in the several tests for their kindness and their patience. Finally, they would like to thank Mr P.R. Persiaux and Ms N.M. Saint-Jean for their valuable remarks.

\section{References}

[1] http://img.arc.nasa.gov/pathfinder/.

[2] http://www.spacemouse.com/.

[3] http://www.spacetec.com/.
[4] R. Aldridge, K. T. Carr, R. England, J. F. Meech, and T. Solomonides. Getting a Grasp on Virtual Reality. In Proc. of the International Conference on Computer Human Interfaces, 1996.

[5] C. Bonnet. Manuel pratique de psychophysique. Armand Colin, Paris, France, 1986.

[6] G. Burdea. Force and Touch Feedback for Virtual Reality. John Wiley and Son, New York, US, 1996.

[7] K. T. Carr and R. England. Simulated and Virtual Realities: Element of Perception. Taylor and Francis Ltd, London, UK, 1995.

[8] R. R. Ellis and S. J. Lederman. The Role of Haptic versus Visual Volume Cues in the Size-Weight Illusion. Perception and Psychophysics, 53(3):315-324, 1993.

[9] G. A. Gescheider. Psychophysics Method, Theory, and Application. Lawrence Erlbaum Associates, New Jersey, US, 1985.

[10] E. B. Goldstein. Sensation and Perception. Brooks/Cole, US, 1999.

[11] G. Hirzinger, B. Brunner, J. Dietrich, and J. Heindl. SensorBased Space Robotics-ROTEX and its Telerobotic Features. IEEE Transactions on Robotics and Automation, 9(5):649663, Oct. 1993.

[12] L. A. Jones and I. W. Hunter. Human Operator Perception of Mechanical Variables and their Effects on Tracking Performance. In Proc. of ASME Winter Annual Meeting: Advances in Robotics, volume 42, pages 49-53, 1992.

[13] M. J. Morgan. Molyneux's Question: Vision, Touch and the Philosophy of Perception. Cambridge University Press, New York, US, 1977.

[14] M. Sams, R. Aulanko, M. Halalainen, M. Hari, O. V. Lounasmaa, S. T. Lu, and J. Simola. Seeing Speech: Visual Information from Lip Movement Modified Activity in the Human Auditory Cortex. Neuroscience letter, 127:5784, 1991.

[15] Sherrick and Cholewiak. A Finite Element Formulation for Nonlinear Incompressible Elastic and Inelastic Analysis. Computers and Structures, 26(1/2):357-409.

[16] M. A. Srinivasan, G. L. Beauregard, and D. L. Brock. The Impact of Visual Information on Haptic Perception of Stiffness in Virtual Environments. In Proc. of ASME Winter Annual Meeting, 1996.

[17] H. Z. Tan, N. I. Durlach, Y. Shao, and M. Wei. Manual Resolution of Compliance when Force and Work Cues are Minimized. In Proc. of ASME Winter Annual Meeting: Advances in Robotics, Mechatronics and Haptic Interfaces, pages 99104, DSC-49, 1993

[18] H. Z. Tan, X. D. Pang, and N. I. Durlach. Manual Resolution of Length, Force, and Compliance. In Proc. of ASME Winter Annual Meeting: Advances in Robotics, volume 42, pages 13-18, 1992

[19] J. Tastevin. En partant de l'expérience d'Aristote. L'Encéphale, 1:57-84, 1937.

[20] C. Tzafestas. Synthèse de retour kinesthésique et perception haptique lors de tâches de manipulation. Ph.D. Thesis, Université de Paris 6, Jul. 1998.

[21] S. Zai. Investigation of Feel for 6dof Inputs: Isometric and Elastic Rate Control for Manipulation in 3D Environments. In Proc. of the Human Factors and Ergonomics Society 37th Annual Meeting, Oct. 1993. 